\title{
Urgences
}

\section{Richard Rohmer, How to Write a Best Seller, Toronto, McClelland and Stewart, 1984, 128 p.}

\section{Gilbert Dupuis}

Numéro 14, août 1986

Corps et jouissances

URI : https://id.erudit.org/iderudit/025270ar

DOI : https://doi.org/10.7202/025270ar

Aller au sommaire du numéro

\section{Éditeur(s)}

Urgences

\section{ISSN}

0226-9554 (imprimé)

1927-3924 (numérique)

Découvrir la revue

Citer ce compte rendu

Dupuis, G. (1986). Compte rendu de [Richard Rohmer, How to Write a Best Seller, Toronto, McClelland and Stewart, 1984, 128 p.] Urgences, (14), 80-81. https://doi.org/10.7202/025270ar d'utilisation que vous pouvez consulter en ligne.

https://apropos.erudit.org/fr/usagers/politique-dutilisation/ 


\section{deliveline}

\section{Richard Rohmer: How to Write a Best Seller, Toron- to, McClelland and Ste- wart, 1984, 128 p.}

\footnotetext{
"Sir, no man but a blockhead ever wrote except for money."
}

- SAMUEL JOHNSON

Selon l'auteur de Periscope Red, Ultimatum, Exxoneration, Exodus! UK, Separation, Balls!, Triad, Retaliation, Patton's Gap, Massacre 747, The Artic Imperative, hors du roman et de l'essai point de best-seller! En neuf ans, Richard Rohmer a écrit neuf best-sellers: sept romans et deux essais. Au Canada, un best-seller appelle des ventes supérieures à 10000 exemplaires...

La planification de l'oeuvre est d'une grande importance. Pour Rohmer, il faut choisir entre la production d'une oeuvre littéraire et celle d'une oeuvre commerciale. Il définit la première comme celle qui véhicule les grands thèmes universels, les personnages forts et les descriptions imagées, le tout with a Iuxuriant prose. L'éventualité du bestseller s'avère mince pour qui choisit cette voie.

L'oeuvre commerciale vise le plus vaste public possible. Elle s'alimente à l'entertainment et à l'intrigue. L'auteur doit se poser la question: "Qui lira ce livre?" Et la réponse devra être: "Des millions de personnes le feront!" Après le choix de la voie populaire, il faudra trouver le concept de l'ouvrage. On ne parle plus de thématique mais de concept. La terminologie du marketing transcende celle de la critique littéraire.

L'idée ou le concept commande la recherche. Les fruits de celle-ci établiront la crédibilité du récit. La documentation des intrigues est essentielle Rohmer favorise l'entrelacement de deux trois intrigues pour prévenir l'ennui du lecteur. Puisque nous écrivons un be\$t-\$eller, ne lésinons pas sur l'investissement: l'embauche d'un ou de plusieurs recherchistes - avec mandat bien défini - peut s'imposer pour sauver du temps à l'artiste... Time is money!, you know!

Puis, l'auteur développera avec soin les intrigues principale et secondaires. On le répète: l'élaboration du plan importe grandement. Les conflits et leurs conséquences constituent la base de toute intrigue. De l'action, beaucoup d'action! L'auteur devra résoudre les problèmes de la chronologie, du point de vue du narrateur, des dialogues, de l'attaque et de la punch line... Comme tout romancier.

On dotera ses personnages de traits facilement reconnaissables. Identification oblige. Rohmer distingue les romans construits autour des personnages et ceux dont l'intrigue domine. Dans la première catégorie, le choix des personnages s'avère capital et l'élaboration de leur psychologie, primordiale. Le pillage des traits de personnes réelles et leurs combinaisons n'arrêtera pas l'écrivain. 
Mais la récupération du voisinage a ses limites: comme Judith Krantz qu'il cite, Rohmer incline à penser que les gens réels sont ennuyeux. exception faite de leurs moments de passion. Qu'il faudrait seuls utiliser.

Le style sera personnel; quelle qu'en soit la venue, se rappeler qu'il charrie deux fonctions: la communication et l'entertainment. On produira la première version - le brouillon - d'instinct, sans autocensure... mais dans le cadre du plan. Rohmer prône l'écriture de plusieurs versions de l'oeuvre. Rewriting. Pour ce faire, un conseil: Show, don't tell! Montrer plutôt que dire. Rewriting.

Le futur écrivain adulé empruntera aux jurons et au langage sexuel avec attention. En assaisonner son récit, n'en pas faire un plat... principal. L'intrigue commandera cette inclusion d'épices. Se rappeler qu'on vise le plus large public possible... Par ailleurs, point de discipline, point de best-seller. La gestion optimale du temps s'impose! Time is money. Les presses toument... Se faire un horaire d'écriture, y tenir! Nul succès sans travail. S'isoler, se concentrer, créer son environnement: Rohmer a un appartement en Foride quand celui de Toronto ne convient pas..

L'éditeur interviendra avant la version finale. Il est votre objectivité. L'éditeur de best-sellers conseille, aide à la recherche, oriente le travail, comige le manuscrit - ne devrait-on pas dire machinescript? Nous sommes loin des imprimeurs qui se prennent pour des éditeurs. La version finale sera le fait d'un travail d'équipe: l'écrivain, les recherchistes, l'éditeur, les lecteurs-conseil... et, bien sûr, l'agent. Futurs écrivains célèbres, il vous faut un agent littéraire, un imprésario, lequel négociera vos contrats, organisera votre promotion et vos entrevues avec les média nationaux, trouvera des débouchés à vos oeuvres: théâtre, cinéma, télévision, conférences... Le best-seller établira le pont entre la littérature et le show business! Et alors les lucarnes lumineuses s'ouvriront pour vous. Et les gens vous verront partout. Ils vous achèteront le lendemain. D'autant plus volontiers que le concept de votre livre stimulera leur consommation des modes...

Au Québec, tous les préalables identifiés par Rohmer sont-ils réunis qui favoriseraient notre part de best-sellers? Que je sache, aucune version québécoise ni française - d'un livre similaire à How to Write a Best Sellern' existe! Existeraitelle qu'elle s'avérerait peut-être une deuxième - hors Le Matou point de salut! - occasion d'éditer un gros vendeur.

Gilbert Dupuis, juin 86

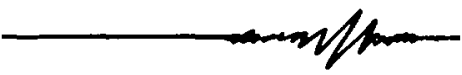

Jean Simard, Félix, Montréal, Stanké, “Québec 10/ 10", no 87 (1947), 1986, $154 \mathrm{p}$.

FÉLIX? Ah oui! Félix Leclerc ou Félix le chat, tout dépendant si notre locuteur est âgé ou jeune. Ah oui! c'est la remise annuelle des trophées Fêlix, nous diront plutôt les mordus de musique, d'arts et spectacles. Or l'amoureux de notre littérature (qu'il soit ou non fin connaisseur ou spécialiste chevronné) saura qu'il s'agit aussi du premier roman publié par Jean Simard en 1947. Jean Simard??? Connais pas! Peut-être, me direz-vous? Ah sacrée mémoire! Et pourtant, cet écrivain, natif de Québec (1916), nous a 